\title{
Crianza parental, sucesos de vida y consumo de drogas en adolescentes escolarizados*
}

\section{Parental Breeding, Life Events and Drug Use in Adolescent Students \\ Criação parental, acontecimentos de vida e consumo de drogas em adolescentes escolarizados}

\author{
Maria Magdalena Alonso Castillo a \\ Universidad Autónoma de Nuevo León, México \\ magdalena_alonso@hotmail.com \\ Verónica Guzmán Ramirez \\ Universidad Autónoma de Tamaulipas, México \\ Nora Angélica Armendáriz García \\ Universidad Autónoma de Nuevo León, México \\ Bertha Alicia Alonso Castillo \\ Universidad Autónoma de Nuevo León, México \\ Nohemi Selene Alarcón Luna \\ Universidad Autónoma de Tamaulipas, México
}

Fecha de recepción: 14 Abril 2016

Fecha de aprobación: 03 Febrero 2017

\section{Resumen:}

El consumo de drogas en los adolescentes es multifactorial, aunque existen factores clave para el desarrollo de esta conducta relacionados con la familia y experiencias de vida. Objetivo: identificar la relación existente entre la percepción de la crianza parental y los sucesos de vida con el consumo de sustancias lícitas e ilícitas en adolescentes. Metodología: el diseño fue descriptivo correlacional, con muestreo probabilístico por conglomerados unietápico. Se obtuvo una muestra de 371 estudiantes de preparatoria de un municipio del estado de Nuevo León, México, donde se consideró un intervalo de confianza del 95\%, una potencia de prueba del $90 \%$, un efecto de diseño de 1,5 y una tasa de no respuesta del 10\%. Para recolectar la información se utilizó una cédula de datos sociodemográficos y de prevalencia de consumo de drogas y la Escala de Estilos Parentales Percibidos (mis memorias de crianza, sucesos de vida) y la Prueba de Identificación de los Trastornos por Uso de Alcohol. La confiabilidad de los instrumentos fue aceptable. Resultados: la crianza parental se relacionó negativa y significativamente con el consumo de alcohol, marihuana y sustancias inhalables. Los sucesos de vida se relacionaron positiva y significativamente con el consumo de alcohol, marihuana, cocaína y sustancias inhalables. Los sucesos de vida expresan el 14\% de la varianza explicada para el consumo de alcohol. Conclusiones: a menor crianza parental, mayor es el consumo de alcohol, marihuana e inhalables; y los sucesos de vida influyen en el consumo de alcohol, marihuana, cocaína y sustancias inhalables.

Palabras clave: adolescente, consumo de alcohol en menores, estrés psicológico, relaciones padre-hijo.

\section{Abstract:}

Drug use in adolescents is multifactorial, there are key factors for the development of this behavior related to family and life experiences. Objective: To identify the relationship between parental breeding perception and life events with licit and illicit substance use in adolescents. Methodology: The design was descriptive correlational, with single-stage probability sampling by conglomerates, obtaining a sample of 371 high school students in a municipality of the State of Nuevo León, México, where it was considered a confidence interval of $95 \%$, a test potency of $90 \%$, a design effect of 1.5 and a non-response rate of $10 \%$. For the data collection a socio-demographic identity card, prevalence of drug use data and the perceived parenting styles scale were used: My Memories of Parenting, Life Events and Proof of Identification Alcohol Use Disorders. The reliability of the instruments was acceptable. Results: Parental breeding was negative and significantly related to alcohol, marihuana and inhalants substances. The life events were positive and significantly related to alcohol, marihuana and inhalants substances. Furthermore, life events express a $14 \%$ of the explained variance for alcohol use. Conclusions: At lower parental breeding, higher alcohol, marihuana and inhalants substances is present; and life events influence alcohol, marihuana, cocaine and inhalants substances.

Keywords: adolescent, underage drinking, stress, psychological, parent-child relations.

Notas de autor 


\section{Resumo:}

O consumo de drogas nos adolescentes é multifactorial, existem fatores finque para o desenvolvimento desta conduta relacionados à família e experiências de vida. Objetivo: identificar a relação que existe entre a percepção da criação parental e os acontecimentos de vida com o consumo de substâncias lícitas e ilícitas em adolescentes. Metodologia: o desenho foi descritivo correlacional, com amostragem probabilístico por conglomerados unietápico, obtendo-se uma mostra de 371 estudantes de preparatoria de um município do Estado de Nuevo León, México, onde se considerou um intervalo de confiança de $95 \%$, uma potência de prova de $90 \%$, um efeito de desenho de 1,5 e uma taxa de não resposta um $10 \%$. Para a coleta de informação utilizou-se uma cédula de dados sociodemográficos e de prevalencia de consumo de drogas, a escala de estilos parentales percebidos: minhas memórias de criação, acontecimentos de vida e a prova de identificação dos transtornos por uso de álcool, a fiabilidade dos instrumentos foi aceitável. Resultados: a criação parental relacionou-se negativa e significativamente com o consumo de álcool, o consumo de maconha, e inhalables. Os acontecimentos de vida relacionaram-se positiva e significativamente com o consumo de álcool, de maconha, cocaína e substâncias inhalables. Ademais os acontecimentos de vida expressam o 14\% da varianza explicada para o consumo de álcool. Conclusões: a menor criação parental, maior é o consumo de álcool, maconha e substâncias inhalables; e os acontecimentos de vida influem no consumo de álcool, maconha, cocaína e substâncias inhalables.

Palavras-chave: adolescente, consumo de álcool por menores, estresse psicológico, relações pais-filho.

\section{Introducción}

Para la disciplina de enfermería, el problema del consumo de sustancias psicoactivas en los adolescentes es de gran relevancia, debido a que su objetivo primordial es brindar cuidado de la salud a personas, familias y comunidades, en el transcurso de su ciclo vital y en los diferentes aspectos de promoción de la salud, prevención de la enfermedad, recuperación y rehabilitación [1]. A partir de esta perspectiva en relación con el consumo de drogas en los adolescentes, el profesional de enfermería debe desarrollar nuevos conocimientos que apoyen la comprensión de este problema multifactorial, a través de una visión teórica de enfermería que pueda brindar, en un futuro, conocimiento base para brindar cuidados específicos a los adolescentes en cuanto a la promoción de la salud y la prevención de consumo de drogas.

En el mundo se ha identificado que el consumo de drogas sigue causando un daño considerable, reflejado en la pérdida de vidas valiosas y años productivos de muchas personas. En el 2012 se informó de un total aproximado de 18.000 muertes relacionadas con las drogas en la población de entre 15 y 64 años de edad. Así mismo, se estima que, en el 2012, entre 162 y 324 millones de personas, es decir, del 3,5\% al 7\% de la población de entre 15 y 64 años, consumieron por lo menos una vez alguna droga ilícita, principalmente sustancias del grupo de cannabis, opioides, cocaína o estimulantes de tipo anfetamínico [2].

En México se ha reportado, por medio de la Encuesta Nacional de Adicciones (ENA) [3], que el consumo de alcohol en los adolescentes aumentó significativamente entre el 2008 y el 2011 . De esta manera, el consumo alguna vez en la vida aumentó del 31,7\% al 42,9\%; en el último año, del 23,7\% al 30\%; y en el último mes, del $11,5 \%$ al $14,5 \%$. Respecto al consumo de tabaco se reporta que la prevalencia de fumadores activos en los adolescentes es del 12,3\%, lo que corresponde a 1,7 millones de adolescentes fumadores. Así mismo, es importante resaltar que el consumo de sustancias ilícitas entre los adolescentes está presente; se ha observado que el consumo de marihuana es el más prevalente alguna vez en la vida $(1,3 \%)$, luego de la cocaína $(0,4 \%)$ y los inhalables $(0,3 \%)$.

En el grupo de adolescentes, el consumo de estas sustancias se ha asociado con problemas de salud, como enfermedades prematuras cardiovasculares, respiratorias y cáncer. Así mismo, se relaciona con diversas problemáticas, como trastornos en la alimentación, conductas sexuales de riesgo, violencia, problemas de aprendizaje y abandono escolar, accidentes automovilísticos, intentos de suicidio, entre otras $[4,5]$.

Además, el inicio o mantenimiento de la conducta de consumo de drogas en los adolescentes se ha asociado con una serie de factores, como tener amigos, hermanos y familiares consumidores de drogas; dificultades en la convivencia familiar, o fracaso en algunas de las funciones o tareas del adolescente que no son afrontadas de manera exitosa, como en el ámbito escolar, social y de incursión laboral. Estos factores pueden potenciarse 
cuando existe un ambiente propicio para adquirir y consumir las sustancias, lo que aumenta la probabilidad de consumo de drogas y disminuye la edad de inicio al consumo [4,6,7].

Uno de los factores que se relacionan con el abuso de sustancias es la percepción que tienen los hijos de la responsabilidad de sus padres para su crecimiento y desarrollo satisfactorios llamada crianza parental. Esta se considerará la percepción del hijo respecto a la responsabilidad que asumen los padres sobre el crecimiento físico, el desarrollo emocional y social de él; este concepto se deriva de la teoría cognitiva social de Bandura e implica una relación recíproca de padres e hijos. Algunos estudios han documentado asociación de la crianza parental y el consumo de drogas licitas o ilícitas $[8,9,10]$.

A su vez, la crianza parental débil en la adolescencia temprana se ha relacionado con la presencia y aumento de eventos estresantes de la vida a lo largo de esta etapa [11]. Los sucesos de vida que vive el adolescente pueden ser potencialmente estresantes y pueden tener un impacto negativo en ellos, los cuales pueden ser afrontados mediante el uso de sustancias adictivas, las que influirán negativamente en su vida futura $[11,12]$. Los sucesos de vida [12] son hechos o acontecimientos que pueden generar estrés y tienen el potencial de desencadenar el consumo de drogas. No obstante, las diferentes respuestas emocionales, cognitivas o del comportamiento que se producen en el adolescente como consecuencia de estos sucesos dependen de la evaluación cognitiva que el adolescente realiza, mediante los factores personales que lo caractericen, así como de su percepción de autoeficacia para poder realizar o no una conducta saludable de salud [13]. Estos sucesos pueden alterar las actividades normales en la vida de los sujetos de manera individual o familiar; acontecimientos o sucesos de la vida como el matrimonio, el nacimiento de hijos, el vivir un divorcio y padecer enfermedades se han relacionado con el consumo de sustancias nocivas y tóxicas [12,14].

El consumo de drogas en los adolescentes es multifactorial. Existen factores clave para el desarrollo de esta conducta asociados con la familia y experiencias de vida; no obstante, se ha identificado un vacío de conocimiento en esta área de promoción de la salud y prevención de las adicciones a las drogas en los adolescentes, incluidos los conceptos de crianza parental, sucesos de vida y consumo de drogas.

Por lo anterior, se establece como propósito identificar la relación que existe entre la percepción de la crianza parental y los sucesos de vida con el consumo de sustancias lícitas (tabaco y alcohol) e ilícitas (marihuana, cocaína e inhalables) en adolescentes que estudian en dos preparatorias de Ciudad Guadalupe, estado de Nuevo León, México. Los resultados del estudio aportan información útil que puede utilizarse en un futuro por el personal de enfermería para el desarrollo y aplicación de intervenciones encaminadas a la promoción de la salud y a la prevención del consumo de sustancias nocivas en adolescentes escolarizados, con énfasis en las diferentes dimensiones de la crianza parental y en el afrontamiento saludable de los sucesos de vida.

\section{Metodología}

El diseño del estudio fue descriptivo correlacional. La población estuvo conformada por 4501 adolescentes pertenecientes a dos preparatorias de una universidad pública. El muestreo utilizado fue probabilístico por conglomerados unietápico y se obtuvo una muestra aleatoria de 371 estudiantes, donde se consideró un intervalo de confianza del $95 \%$, con un error de estimación de 0,05 , una potencia de prueba del $90 \%$, un efecto de diseño de 1,5 y una tasa de no respuesta de un $10 \%$.

Para la recolección de la información se utilizó una cédula de datos sociodemográficos y de prevalencia de consumo de drogas. También se emplearon tres cuestionarios: la Escala de Estilos Parentales Percibidos: Mis Memorias de Crianza (EMBU-I), Sucesos de Vida (SV) y la Prueba de Identificación de los Trastornos por Uso de Alcohol (AUDIT).

La EMBU-I "Mis memorias de crianza" fue la versión de la escala sueca Egna Minnen Betraffande Uppfostran [15] y validada en población mexicana por Márquez-Caraveo y colaboradores [10]. Esta escala evalúa la percepción de crianza parental mediante cuatro dimensiones: apoyo, control, favoritismo y rechazo. Se compone de 82 reactivos, 41 referentes al padre y 41 enfocados en la madre. Los puntajes oscilan de 
82 como puntaje mínimo y 328 como máximo, indicando que a mayor puntaje mayor es la percepción del adolescente sobre la crianza parental. Los coeficientes alfa de Cronbach $(\alpha=0,93$ y $\alpha=0,91)$ obtenidos por los autores en población adolescente de preparatorias han sido satisfactorios [16]. En el presente estudio el alfa de Cronbach fue de 0,89.

El segundo instrumento utilizado fue el cuestionario SV, desarrollado por Lucio y colaboradores [17] para población mexicana. Este identifica la ocurrencia de eventos estresantes experimentados por los adolescentes en un periodo no mayor a un año a través de 130 reactivos que el participante contesta mediante una autoevaluación sobre la experiencia que estos le generaron. El cuestionario está dividido en siete áreas: familiar, social, personal, problemas de conducta, logros y fracasos, salud y escolar. El instrumento se procesa de la siguiente forma: eventos normativos positivos $=1$ punto; eventos normativos negativos $=2$ puntos; eventos no normativos positivos $=3$ puntos; eventos no normativos negativos $=5$ puntos.

La puntuación de los eventos normativos oscila entre 0 y 42 puntos, donde a mayor puntaje el adolescente cuenta con más experiencias que pueden funcionar como factores protectores del bienestar. Por su parte, la puntuación de los eventos no normativos fluctúa entre 0 y 422 puntos, donde a mayor puntaje hay mayor probabilidad de riesgo o alteración del bienestar del sujeto. Villegas y colaboradores [11] obtuvieron una KR-20 de 0,89 con el método de Kuder-Richardson en una muestra de adolescentes de preparatoria; en el presente estudio la confiabilidad fue de 0,90 .

La prueba AUDIT [18], validada para la población mexicana por De la Fuente y Kershenovich [19], evalúa el tipo de consumo de alcohol de los sujetos en los últimos 12 meses, conformada por 10 reactivos, donde los reactivos del 1 al 3 indican la cantidad y la frecuencia de consumo de alcohol; por su parte, los reactivos del 4 al 6 determinan el consumo dependiente. Los reactivos del 7 al 10 evalúan el consumo dañino (considerado perjudicial). Los resultados de los puntajes oscilan de 0 a 40 puntos, entonces, si la calificación es de 1 a 3 se considera consumo sensato (sin riesgo); si es de 4 a 7 , se considera consumo dependiente; y si es de 8 o más puntos, se considera consumo dañino (perjudicial). Este instrumento fue utilizado por Villegas y colegas [11] en adolescentes de preparatorias del área metropolitana de Monterrey, Nuevo León, donde los autores obtuvieron un alfa de Cronbach de 0,81. En este estudio la confiabilidad fue de 0,78.

Antes de la recolección de datos, el estudio fue aprobado por la Comisión de Ética e Investigación, así como por los directivos de las preparatorias donde se llevó a cabo. Se les solicitaron a los directivos las listas de los grupos de estudiantes para la selección aleatoria de estos. Una vez surtido este paso, se les visitó en las aulas para invitarlos a participar, previa explicación de los objetivos de la investigación, así como en lo que constaba su participación en esta.

A los estudiantes que decidieron participar se les entregó el consentimiento informado para los padres, el cual solicita la autorización de estos, y el asentimiento informado del estudiante a ellos mismos, para participar en el estudio. Posteriormente, se estableció una fecha para acudir a cada aula, a efectos de recoger los consentimientos y asentimientos informados, y enseguida proceder al llenado de los instrumentos. Estos se entregaron en un sobre cerrado. También se les reiteró que los datos colectados serían confidenciales y anónimos.

La investigación se desarrolló teniendo en cuenta el marco legal de la Ley General de Salud en Materia de Investigación para la Salud. En esta ley se establece que, para el desarrollo de investigación en salud, el investigador debe apegarse a los aspectos éticos en los que se garantice el respeto a los derechos y el bienestar de los individuos participantes de la investigación [20].

Para el análisis de datos se empleó el paquete estadístico Statistical Package for the Social Sciences (SPSS) versión 20.0; mientras que para el plan de análisis se utilizó estadística descriptiva a través de frecuencias, proporciones, medidas de tendencia central y medidas de dispersión. De igual forma, se utilizó la estadística inferencial. Se determinó la normalidad de la distribución de los datos mediante la prueba de bondad de ajuste de Kolmogorov-Smirnov, con la corrección de Lilliefors, con la cual se decidió el uso de estadísticos no paramétricos. 


\section{Resultados}

La edad que predominó fue 16 años (52,9\%); los datos fueron muy homogéneos para sexo $(51,1 \%$ mujeres vs. $48,9 \%$ hombres $)$, turno $(52,9 \%$ matutino vs. $47,1 \%$ vespertino) y semestre que estaban cursando $(52,9 \%$ para segundo vs. $47,1 \%$ para cuarto). En cuanto a la ocupación, solo el $8,9 \%$ trabaja, y de las ocupaciones que realizaban predominó el ser empleados de servicios como mesero, dependiente o vendedor, repartidor de comida y paquetero. A la vez, se observó que la mayoría de los adolescentes vivía con ambos padres (85,5\%).

También se observó que las sustancias psicoactivas que presentaron en todos los tipos de prevalencias mayores proporciones de consumo fueron alcohol, tabaco y marihuana. Y enseguida de estas sustancias se encontraron cocaína, benzodiacepinas, inhalables y anfetaminas (tabla 1).

TABLA 1

Prevalencias de consumo de drogas en los adolescentes

\begin{tabular}{|c|c|c|c|c|c|c|c|c|c|c|c|c|c|c|c|c|}
\hline \multirow{3}{*}{ Droga } & \multicolumn{16}{|c|}{ Prevalencia } \\
\hline & \multicolumn{2}{|c|}{ Alguna vez en la vida } & \multicolumn{2}{|c|}{$95 \%$ IC } & \multicolumn{2}{|c|}{ En el último año } & \multicolumn{2}{|c|}{$95 \%$ IC } & \multicolumn{2}{|c|}{ En el último mes } & \multicolumn{2}{|c|}{$95 \%$ IC } & \multicolumn{2}{|c|}{ En la última semana } & \multicolumn{2}{|c|}{$95 \%$ IC } \\
\hline & $f$ & $\%$ & Inf & Sup & $f$ & $\%$ & Inf & Sup & $f$ & $\%$ & Inf & Sup & $f$ & $\%$ & Inf & Sup \\
\hline Tabaco & 128 & 33,7 & 29 & 38 & 84 & 22,1 & 18 & 26 & 55 & 14 & 11 & 18 & 46 & 12,1 & 9 & 15 \\
\hline Alcohol & 244 & 64,2 & 59 & 69 & 230 & 60,5 & 56 & 65 & 114 & 30 & 25 & 35 & 62 & 16,3 & 13 & 20 \\
\hline Marihuana & 24 & 6,3 & 4 & 9 & 18 & 4,7 & 3 & 7 & 11 & 2,9 & 1 & 5 & 8 & 2,1 & 1 & 4 \\
\hline Cocaina & 6 & 1,6 & 0 & 3 & 5 & 1,3 & 0 & 2 & 5 & 1,3 & 0 & 2 & 4 & 1,1 & 0 & 2 \\
\hline Benzodiacepinas & 7 & 1,8 & 0 & 3 & 5 & 1,3 & 0 & 2 & 4 & 1,1 & 0 & 2 & 2 & 0,5 & 0 & 1 \\
\hline Anfetaminas & 5 & 1,3 & 0 & 2 & 3 & 0,8 & 0 & 2 & 2 & 0.5 & 0 & 1 & $\ldots$ & $\ldots$ & $\ldots$ & $\ldots$ \\
\hline Inhalables & 8 & 2,1 & 1 & 4 & 4 & 1,1 & 0 & 2 & 2 & 0,5 & 0 & 1 & 1 & 0,3 & 0 & 1 \\
\hline
\end{tabular}

Fuente: Datos propios de estudio

95\% IC: intervalos de confianza del 95\%; Inf: intervalo inferior; Sup: intervalo superior.

En cuanto a la relación entre la crianza parental, los sucesos de vida estresantes y el consumo de drogas, se identificó a través del coeficiente de correlación de Spearman. Se observó que existe una correlación negativa entre la crianza parental y la cantidad de consumo en un día típico de marihuana $\left(r_{s}=-0,120, p<0,01\right)$; así mismo, se encontró una correlación negativa y significativa entre crianza parental y cantidad de consumo en un día típico de inhalables $\left(r_{s}=-0,105 ; p<0,05\right)$. También se observó que la crianza parental $\left(r_{s}=-0,178\right.$; $p<0,05)$ se relacionó negativa y significativamente con el consumo de alcohol y que hay una relación negativa y significativa entre la crianza parental y los sucesos de vida $\left(r_{s}=-0,359 ; p=0,001\right)$.

Aunado a lo anterior, se identificó que los sucesos de vida se relacionaron positiva y significativamente con el consumo de alcohol $\left(r_{s}=0,329 ; p=0,007\right)$ y con la cantidad de consumo de alcohol en un día típico $\left(r_{s}=0,229 ; p=0,001\right)$; a su vez, con la cantidad de consumo de marihuana en un día típico $\left(r_{s}=0,211\right.$; $p=0,001)$, cocaína $\left(r_{s}=0,136 ; p=0,008\right)$ y sustancias inhalables $\left(r_{s}=0,144 ; p=0,005\right)$. Cabe señalar que la correlación positiva y significativa más fuerte de los sucesos de vida fue con el consumo de alcohol. En virtud de este hallazgo, adicionalmente, se consideró el efecto de la crianza parental y los sucesos de vida con el consumo de alcohol (AUDIT).

Para identificar el efecto de la presencia de eventos estresantes y la crianza parental en los adolescentes se empleó un modelo de regresión múltiple, donde los resultados mostraron que únicamente los sucesos de vida estresantes se presentaron como predictores significativos para el consumo de alcohol (puntaje AUDIT) en los adolescentes. De esta manera, los sucesos de la vida estresantes reportan el $14 \%$ de la varianza explicada para el consumo de alcohol (tabla 2). 
TABLA 2

Modelo de regresión lineal múltiple para las variables de crianza parental y sucesos estresantes sobre el consumo de alcohol (AUDIT)

\begin{tabular}{|c|c|c|c|c|c|}
\hline \multicolumn{6}{|c|}{ Modelo $n^{1}=244$} \\
\hline Modelo 1 & df & Suma de Cuadrados & Cuadrado Medio & $\mathbf{F}$ & p \\
\hline Regresión & 1 & 806,96 & 806,96 & 38,53 & 0,001 \\
\hline Residual & 228 & 4774,31 & 20,94 & & \\
\hline \multicolumn{6}{|c|}{$\mathrm{R}_{\text {ajustada }}^{2}=14 \%$} \\
\hline \multicolumn{2}{|c|}{ Variable } & $\beta$ & EE & t & p \\
\hline \multicolumn{2}{|l|}{ EMBU-I } & $-0,041$ & 0,034 & $-1,22$ & 0,250 \\
\hline \multicolumn{2}{|c|}{ Sucesos de vida } & 0.150 & 0,027 & 5,54 & 0,001 \\
\hline
\end{tabular}

Fuente: Datos propios de estudio

df: grados de libertad; EMBU-I: sucesos de vida; EE: error estándar; $\mathrm{n}^{1}$ : consumidores en el último año.

\section{Discusión}

Se documentaron prevalencias de consumo de drogas similares a las reportadas por la ENA [3], donde se destacó el aumento de la prevalencia de consumo de alcohol, lo que indica que es la droga de predilección de los adolescentes.

En cuanto a la prevalencia de consumo de tabaco alguna vez en la vida, se reportó una proporción del 33,7\% de los adolescentes participantes de la investigación, dato superior a lo reportado en la ENA [3], donde la prevalencia global reportada fue del $12,3 \%$ en el país. La prevalencia último año reportada por los adolescentes para el consumo de tabaco fue del 22,1\%; en cuanto a la prevalencia en el último mes, fue del 14,5\% y para la prevalencia en los últimos siete días, fue del 12,1\%.

Para las prevalencias del consumo de alcohol, estas se reportan más altas, con un 64,2\% en la prevalencia alguna vez en la vida. En cuanto a la prevalencia en el último año, fue del 60,5\%; la prevalencia en el último mes fue del 30\%, y para los últimos siete días fue del 16,3\%. Dichas prevalencias son elevadas, tal vez, porque existe la permisibilidad del alcohol dentro de las familias y en los ámbitos de socialización de los adolescentes, en congruencia con diversos autores que han realizado estudios en poblaciones similares [21,22].

Para las drogas ilícitas, las prevalencias globales o alguna vez en la vida fueron más bajas. En el caso de la marihuana, esta presentó una prevalencia del 6,3\%, para sustancias inhalables fue del 2,1\% y para cocaína fue del 1,6\%. Diversos estudios reportaron similitudes en los datos de la prevalencia global de drogas ilícitas; probablemente, en estos adolescentes el vivir con ambos padres y estudiar la preparatoria estén actuando como factores de protección y limitando su involucramiento con estas sustancias tóxicas. Además, pese a que la prevalencia de consumo de drogas ilícitas sea baja, el riesgo de convertirse en poliusuarios de drogas es muy alto, dado el inicio temprano en el consumo de estas sustancias; y de la dependencia y tolerancia de estas sustancias en el organismo.

En esta etapa de transición a la vida adulta es donde se forman y consolidan los estilos de vida futuros, que pueden seguir reproduciéndose en etapas posteriores y deteriorar su vida y sus relaciones sociales y ocasionar un gran sufrimiento familiar. Algunos autores también explican la experimentación de consumo de drogas, porque los adolescentes pueden estar buscando nuevas emociones, imitando comportamientos de amigos o buscando la aceptación de ciertos grupos [23,24].

En cuanto a la relación de la crianza parental con el consumo de alcohol, tabaco, marihuana, cocaína e inhalables, se encontraron correlaciones negativas significativas con la cantidad de consumo en un día típico de marihuana y sustancias inhalables, así como con el consumo de alcohol (AUDIT). Esto coincide con Villegas y colaboradores [16] y Laghi y colaboradores [25], quienes documentaron que la percepción negativa 
de la crianza parental se asocia al inicio o incremento del consumo de drogas. Estos resultados concuerdan teóricamente con algunos conceptos del modelo de Nola J. Pender [26,27], donde se observa que los factores socioculturales, como la percepción positiva de la crianza parental, promueven la conductas de rechazo asertivas ante la invitación o la presión de los pares para que consuman drogas, probablemente porque en la familia la crianza de sus padres los dota de habilidades sociales, de comunicación parental, y les otorga la confianza en sí mismos, que potencializa la presencia de una conducta saludable, que en este caso es la disminución del consumo de alcohol.

Además, en este estudio se encontró una relación negativa y significativa de la crianza parental con los sucesos de vida estresantes. Desde la perspectiva de Pender, estos resultados se explican mediante su modelo, donde se observa que los factores socioculturales y las influencias situacionales se encuentran relacionados e interactúan entre sí sobre la percepción de la autoeficacia, y a partir de estos se puede o no lograr la conducta saludable de salud, es decir, una crianza parental débil en la adolescencia temprana se asocia con los sucesos de vida estresantes en la adolescencia, lo que a su vez se relaciona con el consumo de drogas, mediado tal vez por el déficit de habilidades de asertividad en los adolescentes que consumen drogas en esta etapa. Probablemente esto ocurre en aquellos que no perciben una crianza parental fuerte y efectiva.

Así mismo, se documentó una correlación positiva y significativa de los sucesos de vida estresantes y la cantidad de consumo de alcohol, marihuana, cocaína y sustancias inhalables; al igual que con el consumo de alcohol evaluado por el AUDIT. Esto es similar a lo mostrado por otros autores [11,28], quienes indican que a mayor número de sucesos de vida estresantes y el mayor tiempo de duración de estos eventos, más importante es el consumo de alcohol y de otras drogas.

Aunado a lo anterior, se observó en este estudio que los sucesos de vida estresantes son predictores significativos del consumo de alcohol. Tal observación apoya lo propuesto por Pender, donde los factores personales afectan las influencias situacionales, y con base en estas el individuo es capaz o no de comprometerse a la realización de algún cambio en su estilo de vida; sin embargo, cuando no existe previamente esa concientización de dicho compromiso, no se cumple con el cambio o mantenimiento de conducta saludable.

\section{Conclusiones}

Se identificó una relación negativa y significativa entre la percepción de la crianza parental y la cantidad de consumo de marihuana, inhalables y alcohol en un día típico. Así mismo, se observó una correlación positiva y significativa entre la presencia de sucesos de vida estresantes con la cantidad de consumo de alcohol, marihuana, cocaína y sustancias inhalables en un día típico.

Estos hallazgos pueden ser útiles para fortalecer factores protectores, como la crianza parental, para prevenir el consumo de drogas mediante intervenciones de enfermería aplicadas en adolescentes y sus padres.

Conflicto de intereses

Ninguno de los autores manifiesta conflicto para la publicación de este manuscrito.

\section{Referencias}

1. United Nations Office on Drugs and Crime. World Drug Report 2014 [internet]; 2014 [citado 2016 ene 2]. Disponible en: https://www.unodc.org/documents/lpo-brazil//noticias/2014/06/World_Drug_Report_201 4_web_embargoed.pdf 
2. Oficina de las Naciones Unidas contra la Droga y el Delito. Abuso de drogas en adolescentes y jóvenes y vulnerabilidad familiar [internet]; 2013 [citado 2016 ene 02]. Disponible en: https://www.unodc.org/docum ents/peruandecuador

3. Instituto Nacional de Psiquiatría Ramón de la Fuente Muñiz, Instituto Nacional de Salud Pública, Secretaría de Salud. Encuesta Nacional de Adicciones 2011: Reporte de drogas. [internet]; México; 2011 [citado 2016 Ene 03]. Disponible en: http://www.conadic.salud.gob.mx/pdfs/ENA_2011_DROGAS_ILICITAS_.pdf

4. Organización de los Estados Americanos. El problema de las drogas en las Américas. Washington: OEA; 2012.

5. Secretaría de Salud de México. Actualidad en adicciones: consumo de alcohol y tabaco en México [internet]; 2012 [citado 2016 ene 4]. Disponible en: http://www.conadic.salud.gob.mx/pdfs/libro4.pdf

6. Morera JAC, Noh S, Hamilton H, Brands B, Gastaldo D, Wright MGM. Factores socioculturales y consumo de drogas entre estudiantes universitarios costarricenses. Texto Contexto Enferm [internet]. 2015 [citado 2016 ene 3];24(especial):145-53. Disponible en: http://www.scielo.br/scielo.php?script=sci_arttext\&pid=S0104-0707 2015000600145\&lng=en.http://dx.doi.org/10.1590/0104-07072015001170014.

7. Lerma-Cabrera JM, Steinebach P, Carvajal F, Ulloa B, Cid-Parra C, Langer AI. Factores de riesgo asociados al consumo problemático al alcohol en adolescencia: el rol preventivo de Mindfullnes. Rev Psy Soc Educ. 2015;7(1):57-69.

8. Bandura A. Social foundations of thought and action: A social-cognitive theory. Englewood Cliffs, NJ: Prentice Hall; 1986.

9. Florenzano UR, Valdés CM, Cáceres CE, Casassus RM, Sandoval IA, Santander RS, et al. Percepción de la relación parental entre adolescentes mayores y menores de 15 años. Rev Chi Pediatr. 2009;80(6):509-27.

10. Márquez ME, Hernández L, Villalobos J, Pérez V, Reyes M. Datos psicométricos del EMBU-I “Mis memorias de crianza" como indicador de la percepción de crianza en una muestra de adolescentes de la Ciudad de México. Rev Salud Mental. 2007;30(2):58-66.

11. Villegas PMA, Alonso MM, Alonso BA, Guzmán FFR. Eventos estresantes y la relación con el consumo de alcohol y tabaco en adolescentes. Rev Cienc Enferm. 2014;20(1):35-46.

12. Holmes T, Rahe RH. The social readjustment rating scale. J Psychosom Res. 1967;11(1):213-8.

13. Lagoueyte Gómez MI. El cuidado de enfermería a los grupos humanos. Rev Univ Ind Santander Salud. 2015;47(2):209-13.

14. Díaz K, Amaya MCP. Factores familiares individuales y ambientales y no consumo de drogas en adolescentes. Rev Av Enferm. 2012;30(3):37-59.

15. Perris C, Jacobsson L, Lindström H, Von Knorring L, Perris H. Development of a new inventory for assessing memories of parental rearing behaviour. Acta Psychiatr Scand. 1980;61(1):265-74.

16. Villegas-Pantoja M, Alonso-Castillo M, Alonso-Castillo B, Martínez-Maldonado R. Percepción de crianza parental y su relación con el inicio del consumo de drogas en adolescentes mexicanos. Aquichan. 2014;14(1):41-52.

17. Lucio GM, León I, Durán C, Bravo E, Velasco E. Los sucesos de vida en dos grupos de adolescentes de diferente nivel socioeconómico. Salud Mental. 2001;24(5):17-24.

18. Babor TF, Higgins-Biddle JC, Saunders J, Monteiro M. Cuestionario de identificación de los trastornos debido al consumo de alcohol [internet]. Geneva: Organización Mundial de la Salud; 2001 [citado 2016 ene 21]. Disponible en: http://www.who.int/substance_abuse/activities/en/AUDITmanualSpanish.pdf

19. De la Fuente J, Kershenovich D. El alcoholismo como problema médico. Rev Med UNAM. 1992;35(2):47-51.

20. Secretaría de Salud de México. Reglamento de la Ley General de Salud en materia de Investigación para la Salud [internet]; 1987. Disponible en: http://www.salud.gob.mx/unidades/cdi/nom/compi/rlgsmis.html

21. Telumbre-Terrero J, Sánchez-Jaimes B. Consumo de alcohol en adolescentes del estado de Guerrero, México. HAAJ [internet]. 2015 [citado 2016 abr 6];15(1):79. Disponible en: http://ojs.haaj.org/index.php/haaj/article/view $/ 217$

22. Jones $S$, Magee $C$. The role of family, friends and peers in Australian adolescent's alcohol consumption. Drug Alcohol Rev. 2014;33(3):304-13. doi: 10.1111/dar.12111 
23. Subin P, Yeni K, Park S, Kim Y. Prevalence, correlates, and associated psychological problems of substance use in Korean adolescents. BMC Public Health. 2016;16:79. doi: 10.1186/s12889-016-2731-8

24. Khan M, Cleland C, Scheidell J, Berger A. Gender and racial/ethnic differences in patterns of adolescent alcohol use and associations with adolescent and adult illicit drug use. Am J Drug Alcohol Abuse. 2014;40(3):213-24.

25. Laghi F, Lonigro A, Baiocco R, Baumgartner E. The role of parenting styles and alcohol expectancies in teen binge drinking: A preliminary investigation among Italian adolescents and their parents. Drug Educ Prev Polic. 2013;20(2):131-9.

26. Aristizábal HGP, Blanco BDM, Sánchez RA, Ostiguín MRM (2011). El modelo de promoción de la salud de Nola Pender: una reflexión en torno a su comprensión. Enferm Univ. 2011;8(4):16-23.

27. Pender NJ, Pender AR. Illness prevention and health promotion services provided by nurse practitioners: predicting potential consumers. Am J Public Health. 1980;70(8):798-803

28. Pérez M, Lucio-Gómez E. Construcción de un modelo de riesgo en el consumo de alcohol y otras sustancias ilícitas en adolescentes estudiantes de bachillerato. HAAJ [internet]. 2010 [citado $2016 \mathrm{abr}$ 7];10(1): 9-96. Disponible en: http://www.biblioteca.cij.gob.mx/Archivos/Materiales_de_consulta/Drogas_de_Abuso/Artic ulos/Construcciondeunmodeloderiesgoconsumodealcoholyosustancias.pdf

\section{Notas}

* Artículo de investigación.

\section{BY}

\section{Información adicional}

Cómo citar: Alonso Castillo MM, Guzmán Ramírez V, Armendáriz García NA, Alonso Castillo BA, Alarcón Luna NS. Crianza parental, sucesos de vida y consumo de drogas en adolescentes escolarizados. Investig Enferm Imagen Desarr. 2018;20(1):xx-xx. https://doi.org/10.11144/Javeriana.ie20-1.cpsv 